\title{
Influence of perturbations on period-doubling bifurcation
}

\section{Svensmark, Henrik; Samuelsen, Mogens Rugholm}

\section{Published in:}

Physical Review A

Link to article, DOI:

10.1103/PhysRevA.36.2413

Publication date:

1987

\section{Document Version}

Publisher's PDF, also known as Version of record

Link back to DTU Orbit

Citation (APA):

Svensmark, H., \& Samuelsen, M. R. (1987). Influence of perturbations on period-doubling bifurcation. Physical Review A, 36(5), 2413-2417. https://doi.org/10.1103/PhysRevA.36.2413

\section{General rights}

Copyright and moral rights for the publications made accessible in the public portal are retained by the authors and/or other copyright owners and it is a condition of accessing publications that users recognise and abide by the legal requirements associated with these rights.

- Users may download and print one copy of any publication from the public portal for the purpose of private study or research.

- You may not further distribute the material or use it for any profit-making activity or commercial gain

- You may freely distribute the URL identifying the publication in the public portal

If you believe that this document breaches copyright please contact us providing details, and we will remove access to the work immediately and investigate your claim 


\title{
Influence of perturbations on period-doubling bifurcation
}

\author{
H. Svensmark and M. R. Samuelsen \\ Physics Laboratory I, The Technical University of Denmark, DK-2800 Lyngby, Denmark
}

(Received 12 December 1986)

\begin{abstract}
The influence of noise and resonant perturbation on a dynamical system in the vicinity of a period-doubling bifurcation is investigated. It is found that the qualitative dynamics can be revealed by simple considerations of the Poincare map. These considerations lead to a shift of the bifurcation point which is proportional to the square of the amplitude of the perturbation. The results of this investigation are in agreement with numerical calculations for the microwave-driven Josephson junction.
\end{abstract}

\section{INTRODUCTION}

Dynamical systems which undergo periodic motion are of great theoretical interest and also of importance for applications. Not only the existence of periodic orbits but also the structural stability of these is important. In this paper we focus on the stability of a periodic orbit against period-doubling bifurcation in the presence of noise and a small resonant signal, a problem which has attracted considerable interest. ${ }^{1-6}$ It is shown that the qualitative dynamics in the vicinity of a period-doubling bifurcation can be derived from very simple considerations and that these results are in qualitative agreement with previous results. ${ }^{1-6}$ These considerations differ from the previous ones $^{1-3}$ in the way that the dynamics is investigated exclusively on the basis of the Poincare map. Furthermore, the investigation takes its origin in the fix points in the Poincaré map and can therefore be based on linearstability analysis. The results show that the shift of the bifurcations point is proportional to the square of the perturbating amplitude, a result which is confirmed by experiments on Josephson junctions. ${ }^{7}$ As an example of the influence of perturbations on a period-doubling bifurcation, a numerical study of the microwave-driven Josephson junction is reported.

We consider a dissipative dynamical system modeled by the following differential equation:

$\dot{x}=f_{\mu}(x, t)+a(t)+s(t), \quad(x, t) \in \mathbb{R}^{n} \times \mathbb{R}, \quad \mu \in \mathrm{I}$.

Here $f(\cdot, t)=f(\cdot, t+T)$ is periodic in $t$ with period $T, \mu$ is a control parameter defined in some interval $I$, $a(t)=a(t+2 T)$ is periodic in $t$ with period $2 T$, and $s(t)$ is a white-noise term defined by $\langle s(t) s(t+\tau)\rangle=\sigma \delta(\tau)$ and $\langle s(t)\rangle=0 . \quad a(t)$ and $s(t)$ are small compared to $f(x, t)$, i.e., the perturbations are small. Let us first set these perturbations to zero. As the control parameter $\mu$ is varied, changes in the structure of the asymptotically stable solution governed by Eq. (1) may occur. In this paper it is assumed that this solution changes from a periodic orbit $\gamma$ of period $T$ to a periodic orbit $\gamma^{*}$ of period $2 T$.

\section{DYNAMICS IN THE POINCARÉ MAP}

A tool for investigation of the asymptotic behavior of orbits close to a closed orbit is the Poincare map. ${ }^{8}$ Here this map is given by a local transversal section of the orbit in phase space $\psi(x, t)$, at integer numbers of period $T$, $\psi(x, n T)=\psi_{T}^{n}(x)$, and is written $P\left(x_{0}\right)=\psi_{T}\left(x_{0}\right)$. With the aid of the Poincare map one can derive a discrete dynamical system arising from Eq. (1),

$$
x_{n+1}=P_{\mu}\left(x_{n}\right), \quad x \in \mathbb{R}^{n} .
$$

A fix point of the discrete map Eq. (2) corresponds to a closed orbit of period $T$, and it is clear that the stability of the fix point reflects the stability of the closed orbit. A period-doubling bifurcation is associated with an eigenvalue $\lambda=-1$ at the fix point $x^{*}$ of the Poincaré map Eq. (2). This means that an orbit $\gamma^{*}$ of the orbit $\psi(x, t)$ alternates from one side of the fix point $x^{*}$ to the other along the direction of the eigenvector $e_{\lambda}$ for $\lambda=-1$. Orbits like $\gamma^{*}$ are confined on a two-dimensional surface called the center manifold. ${ }^{8}$ This surface can be descrbed as a Möbius band, i.e., a band with a half twist in it (see Fig. 1). The asymptotic behavior of the orbits near a fix point, in the vicinity of a period-doubling bifurcation, can be revealed by linearization of the discrete map around the fix point. Since the asymptotic behavior in the directions of the other eigenvectors is relaxing fast, the asymptotic behavior can be described exclusively with respect to the direction of the eigenvector for $\lambda=-1$. This means that the discrete map will be one dimensional (see Fig. 1), regardless of the original dimension of the dynamical system. ${ }^{8}$ It should be noted that this way of describing the asymptotic behavior agrees with the previous description. ${ }^{1-4}$ Instead of the control parameter $\mu$ the eigenvalue $\lambda$ of the discrete map will be used, assuming that $\mu$ and $\lambda$ are linearly related in a narrow region close to the bifurcation. So linearizing Eq. (2) around the fix point, and using the mentioned assumptions, the equation describing the asymptotic behavior becomes

$$
\xi_{n+1}=\lambda \xi_{n}, \quad \xi \in \mathbb{R} .
$$

$\xi$ is the deviation from the fix point in the direction of the eigenvector for $\lambda \simeq-1$, if $\lambda>-1$ the fix point is stable, but if $\lambda<-1$ the fix point has become unstable and a period-doubling bifurcation has occurred. Higherorder terms in $\xi_{n}$ are not necessary since this is a linear stability analysis.

If we now look at the influence of a small resonant per- 


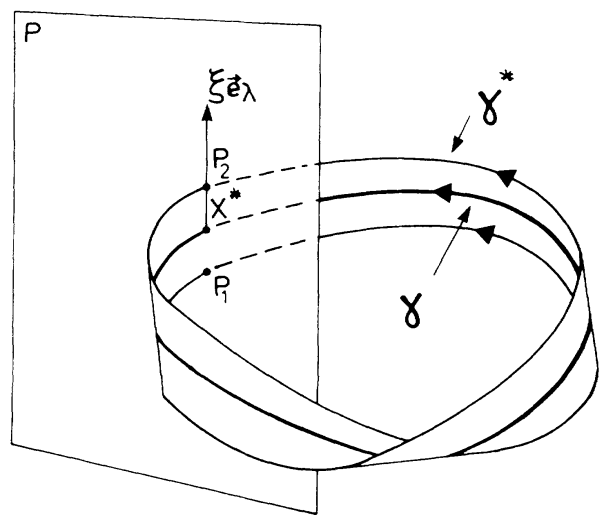

FIG. 1. Intersection of a two-dimensional manifold with the Poincare map $P$. The periodic orbit $\gamma$ of period $T$ is confined to this manifold and intersects $P$ at the fix point $x^{*}$. The vector $e_{\lambda}$ in $P$ is the eigenvector for the eigenvalue $\lambda=-1 . \gamma^{*}$ is a periodic orbit of period $2 T$ which intersects $P$ at the points $p_{1}$ and $p_{2}$.

turbation at half of the fundamental frequency $[a(t) \neq 0$ in Eq. (1)], the discrete map Eq. (2) will, of course, be changed by the perturbation. The change in the discrete map Eq. (2) is written

$$
x_{n+1}=P_{n}^{*}\left(x_{n}\right), \text { where } P_{n+2}^{*}(\cdot)=P_{n}^{*}(\cdot)
$$

since the driving terms in Eq. (1) now have the periodicity $2 T$. The function $P_{n}^{*}\left(x_{n}\right)$ is approximated in the following way:

$$
P_{n}^{*}\left(x_{n}\right)=P\left(x_{n}\right)+P_{n}^{\prime}\left(x_{n}\right), \quad P_{n}^{\prime}(\cdot)=P_{n+2}^{\prime}(\cdot),
$$

where $P\left(x_{n}\right)$ is the unperturbed Poincare map and $P_{n}^{\prime}\left(x_{n}\right)$ is a small perturbation with the given periodicity. Linearizing around the fix point $x^{*}$ gives

$$
\xi_{n+1}=\lambda \xi_{n}+(-1)^{n} A_{1} \xi_{n}+(-1)^{n} A_{0},
$$

where the last two terms are part of an expansion of $P_{n}(x)$. It is assumed that this expansion of $P_{n}$ is the simplest possible with the given periodicity. In the expansion, terms such as $B_{0}$ and $B \xi_{n}$ are neglected since they correspond to resonant terms at the driving frequency and not on half the fundamental frequency. $A_{1}$ and $A_{0}$ are small compared with 1 . Since the perturbed Poincare map has the periodicity of 2 , it is natural to seek fix points with this periodicity,

$$
\xi_{n+2}=\left(\lambda^{2}-A_{1}^{2}\right) \xi_{n}+\left[\lambda-(-1)^{n} A_{1}-1\right](-1)^{n} A_{0} .
$$

Inserting the fix point $\xi_{n}^{*}$ one gets

$$
\xi_{n}^{*}=\frac{(-1)^{n} A_{0}\left[\lambda-(-1)^{n} A_{1}-1\right]}{1-\left(\lambda^{2}-A_{1}^{2}\right)}
$$

or

$$
\xi_{\mathrm{odd}}^{*}-\xi_{\mathrm{even}}^{*}=\frac{2 A_{0}(1-\lambda)}{1-\left(\lambda^{2}-A_{1}^{2}\right)} .
$$

These fix points correspond to intersection of the orbit at either even or odd numbers of the period $T$, that is, $n$ ei- ther even or odd. From this result one observes two things. First the influence of the resonant perturbation is to stabilize the system against bifurcation as seen from Eq. (7), where the squared eigenvalue is reduced by $A_{1}^{2} \cdot{ }^{3}$ This leads to a bifurcation shift which is proportional to $A_{1}^{2}$. Experiments on Josephson junctions, where the detuning is very small, confirms this result. ${ }^{7}$ Second, as seen from Eq. (8), the small perturbation is amplified as the reduced eigenvalue tends to $-1 .^{2}$

The influence of noise alone on the asymptotic behavior can be modeled by adding a small stocastic term $s_{n}$ to Eq. (3) so this becomes

$$
\xi_{n+1}=\lambda \xi_{n}+s_{n},
$$

where $s_{n}$ has the following properties; $\left\langle s_{n}\right\rangle=0$ and $\left\langle s_{n} s_{m}\right\rangle=\sigma \delta_{n m}$, the bracket means ensemble averaging and $\delta_{n m}$ Kroneckers delta. $s_{n}$ describes the random intersection of orbits in the Poincare map along the direction of the eigenvector $e_{\lambda}$ for $\lambda=-1$, caused by the noise. Equation (9) can be solved ( $\lambda^{n} \rightarrow 0$ for $\left.n \rightarrow \infty\right)$,

$$
\xi_{n+1}=\sum_{i=0}^{\infty} s_{n-i} \lambda^{i}
$$

The ensemble average of $\xi_{n}$ is

$$
\left\langle\xi_{n}\right\rangle=0
$$

since $\left\langle s_{n}\right\rangle=0$ and the autocorrelation function is

$$
\left\langle\xi_{n} \xi_{n+m}\right\rangle=\frac{\lambda^{|m|}\left\langle s_{n}^{2}\right\rangle}{1-\lambda^{2}} .
$$

This result shows that the noise is amplified as the eigenvalue $\lambda$ approaches -1 . Since $\lambda$ is negative $\xi$ relaxes towards the fix point in an alternating fashion, centering the noise spectrum about half of the fundamental frequency. ${ }^{4}$

Finally, the influence of both noise and a small resonant perturbation is investigated $[a(t) \neq 0$ and $s(t) \neq 0$ in Eq. (1)]. By Eqs. (6) and (9) the equation describing the asymptotic behavior becomes

$$
\xi_{n+1}=\lambda \xi_{n}+(-1)^{n} A_{1} \xi_{n}+(-1)^{n} A_{0}+s_{n} .
$$

Using the same procedure as in Eqs. (6) and (9) the average value of $\xi_{n}$ becomes as expected the same as Eq. (8a), and the autocorrelation function

$$
\begin{aligned}
\left\langle\left(\xi_{n}-\left\langle\xi_{n}\right\rangle\right)\left(\xi_{n+2 j}-\left\langle\xi_{n}\right\rangle\right)\right\rangle & \\
= & \frac{\left\{1+\left[\lambda-(-1)^{n} A_{1}\right]^{2}\right\}\left(\lambda^{2}-A_{1}^{2}\right)^{|j|}\left\langle s_{n}^{2}\right\rangle}{1-\left(\lambda^{2}-A_{1}^{2}\right)^{2}} .
\end{aligned}
$$

It is seen that the general features are the same as when the perturbations were added separately, that is, the amplification of both the noise and the small signal and a reduction of the critical eigenvalue, i.e., a stabilization against the bifurcation. It should be noted that it is also possible to see the stabilization against the bifurcation in the case of a near-resonant signal which is different from half of the fundamental frequency. This is done in the same way as before, but now the function $P_{n}^{\prime}\left(x_{n}\right)$ has a periodicity which is much larger than 2, see Eq. (5). 


\section{NUMERICAL CALCULATIONS}

In order to illustrate the features of the above theory, the differential equation describing a microwave-driven Josephson junction is integrated numerically. The governing differential equation is ${ }^{9}$

$$
\phi_{t t}+\alpha \phi_{t}+\sin \phi=A_{D} \sin \left(\omega_{D} t\right)+A_{s} \sin \left(\omega_{s} t\right)+\eta+n(t) .
$$

Here $\phi$ is the quantum-mechanical phase difference across the junction and $\alpha$ is a damping parameter. $A_{D}$ and $A_{s}$ are the driving and resonant perturbing amplitudes normalized to the critical current of the junction $I_{0}, \omega_{D}$, and $\omega_{s}$ are the corresponding frequencies normalized to the maximum plasma frequency of the junction. $\eta$ is the normalized bias current and $n(t)$ is a noise current to be specified below. We note that Eq. (14) also describes a driven damped pendulum.

In the numerical calculations described here Eq. (14) is integrated using a fourth-order Runge-Kutta method with 32 points per period of the drive. 256 drive periods are integrated and the first 128 are discarded to remove transients. The noise is assumed to have a white spectrum described by the parameter $\Gamma=2 e k T / \hbar I_{0}$, i.e., the ratio of the thermal energy $(k T)$ to the Josephson coupling energy $(\hbar / 2 e) I_{0}$. Typical experimental values for Josephson junctions correspond to $\Gamma=10^{-4}-10^{-3}$. In the calculations the white noise is constructed using a randomnumber generator. Throughout the calculations the following values of the parameters were used: $\alpha=0.2$, $A_{D}=0.85, A_{s}=0$ and $0.001, \omega_{D}=1.6$ and $\omega_{s}=0.8 . \eta$ and $n(t)$ were varied. The parameters were chosen so the system would get near a period-doubling bifurcation with the variation of $\eta$, here used as control parameter.

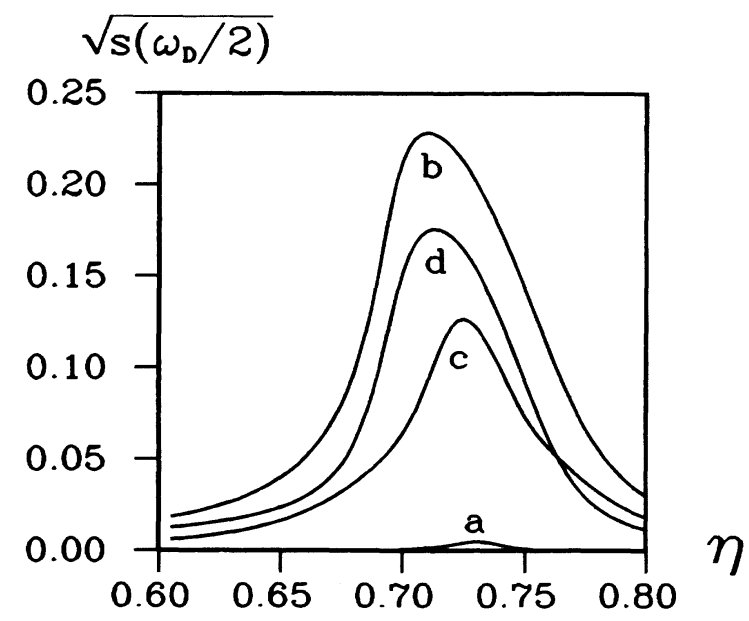

FIG. 2. The amplitude of the response at the signal frequency $\omega_{s}=0.8$ for $A_{D}=0.85, \omega_{D}=1.6$ vs $\eta$. Curve $a$, without any perturbations $A_{s}=0$ and $\Gamma=0$. Curve $b$, with a small resonant perturbation, $A_{s}=0.001, \omega_{s}=0.8$, and $\Gamma=0$. Curve $c$, with only noise added, $A_{s}=0$ and $\Gamma=10^{-4}$. Curve $d$, where both noise and a resonant perturbation are present, $A_{s}=0.001$, $\omega_{s}=0.8$, and $\Gamma=10^{-4}$.
Figure 2 illustrates for $A_{D}=0.85$ and $\omega_{D}=1.6$ an example of the influence of noise and a small resonant signal in the vicinity of a period-doubling bifurcation. Here the amplitude of the response at the signal frequency $\omega_{s}=0.8$ is plotted versus the control parameter $\eta$, which is the normalized bias current. Figure 2 (a) shows the amplitude in the absence of both noise and signal $A_{s}=0$ and $n(t)=0$ (this response is in fact due to the extremely slow relaxation). In Fig. 2(b) a small signal is included ( $A_{s}=0.001, \omega_{s}=0.8$ ) and it is seen that the small signal is amplified in the vicinity of the period-doubling bifurcation in agreement with the above theory. In Fig. 2(c) the signal is absent but now noise is included $\left(\Gamma=10^{-4}\right)$, and it is seen that the noise is amplified in the vicinity of the bifurcation. In Fig. 2(d) both noise and signal are included $\left(A_{s}=0.001\right.$ and $\left.\omega_{s}=0.8\right)$ and again the amplification of the perturbations is seen.

One way of characterizing the stability of a dynamical system against a period-doubling bifurcation is to calculate the maximum Liapunov exponent. ${ }^{10}$ When this exponent is negative the periodic orbit of period $T$ is stable, but when the exponent tends to zero the orbit gets unstable against an orbit of period $2 T$. Figure 3 illustrates the maximum Liapunov exponent $\sigma_{\max }$ versus the control parameter $\eta$. Figure 3(a) shows the maximum Liapunov exponent in the absence of a small signal and noise ( $A_{s}=0$ and $\Gamma=0$ ) where it is seen that the exponent comes very close to zero, i.e., to a period-doubling bifurcation. In Fig. 3(b) a small signal is included ( $A_{s}=0.001$ and $\Gamma=0$ ) and it is seen that the exponent is reduced in the vicinity of the bifurcation showing the stabilization of the system against bifurcation in agreement with the above theory. Figures 3(c) and 3(d) are similar to Figs. 3(a) and 3(b) but here noise is included $\left(\Gamma=10^{-4}\right)$. In Fig. 3(c) only minor effects of the noise are seen, whereas the presence of noise

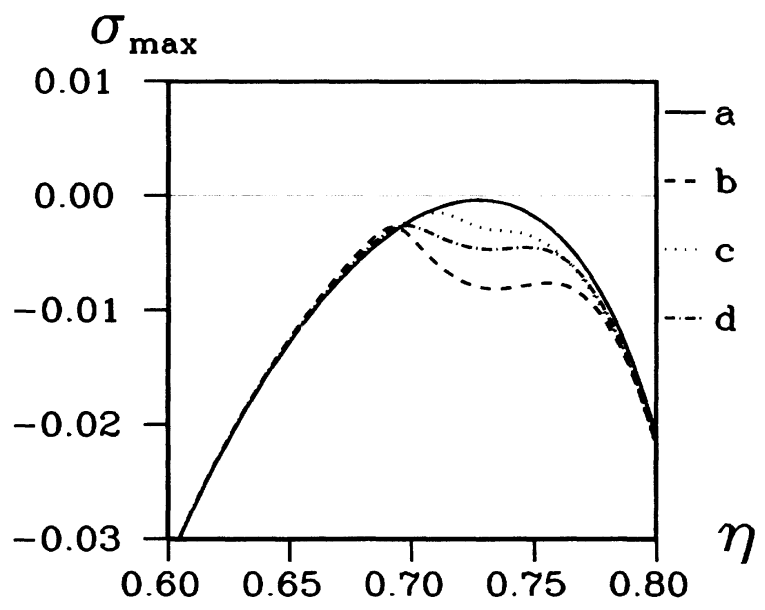

FIG. 3. Maximum Liapunov exponent $\sigma_{\max }$ vs $\eta$, for $A_{D}=0.85, \omega_{D}=1.6$. Curve $a$, without resonant perturbation and noise, $A_{s}=0$ and $\Gamma=0$. Curve $b$, with resonant perturbation and no noise $A_{s}=0.001, \omega_{s}=0.8$, and $\Gamma=0$. Curve $c$, with noise but no resonant perturbation, $\Gamma=10^{-4}$ and $A_{s}=0$. Curve $d$, with noise and resonant perturbation, $A_{s}=0.001, \omega_{s}=0.8$, and $\Gamma=10^{-4}$. 

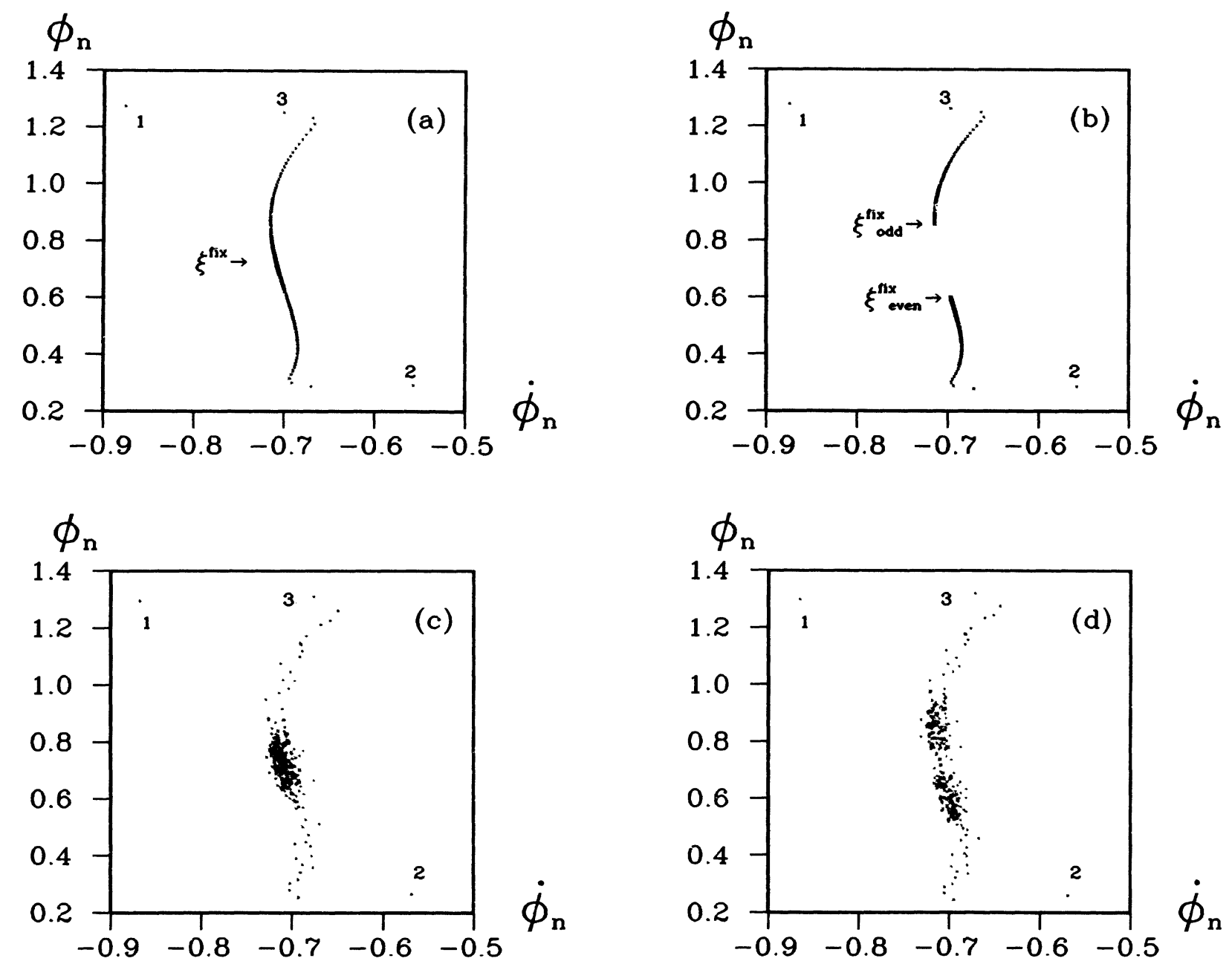

FIG. 4. Asymptotic behavior around the fix point in the Poincare map. The numbers 1,2,3, indicate the successive intersection of orbits with the Poincare map. $A_{D}=0.85, \omega_{D}=1.6$ and $\eta=0.68$. (a) Without perturbations, $A_{s}=0$ and $\Gamma=0$. (b) With a resonant perturbation, $\boldsymbol{A}_{s}=0.001, \omega_{s}=0.8$, and $\Gamma=0$. (c) Only noise is present, $\boldsymbol{A}_{s}=0$ and $\Gamma=10^{-4}$. (d) Both noise and a resonant perturbation are present, $A_{s}=0.001, \omega_{s}=0.8$, and $\Gamma=10^{-4}$.

and signal in Fig. 3(d) reduces the exponent in the vicinity of bifurcation.

In the above theory it was assumed that the asymptotic behavior was confined to a line in the Poincare map; in order to illustrate this numerically, successive intersections of orbits with the Poincare map were calculated. Figure 4 shows the relaxation towards the fix point in the Poincare map for $\eta=0.68$. The relaxation is very fast in the direction perpendicular to the curve on which the essential asymptotic behavior is confined. The numbers $(1,2,3$,$) in Fig. 4$ indicate the alternating relaxation towards fix point(s) or steady state in the presence of noise. It is seen that the asymptotic behavior, with no noise added, is strictly confined to a one-dimensional curve in the Poincaré map [see Figs. 4(a) and 4(b)], and so confirming that the asymptotic behavior in the full phase space is confined on a two-dimensional manifold. In the presence of noise the asymptotic behavior is not as strictly confined to the one-dimensional curve in the Poincare map [see Figs. 4(c) and 4(d)]. The asymptotic behavior shown in Fig. 4 agrees with the basic postulates of the present and the previous theory. ${ }^{1-4}$

As a result of Fig. 4, where only very small perturbations are used $\left(A_{s}=0.001\right)$, the dynamics close to a period-doubling bifurcation in the presence of larger perturbations (e.g., $A_{s}=0.01$ ), ${ }^{5}$ have to be described by a two-dimensional manifold in the full phase space, where the curvature of the manifold has to be considered (the Poincaré section is no longer a line but a curve).

\section{CONCLUSION}

In conclusion it has been shown that the influence of perturbations on the qualitative dynamics of a system in the vicinity of a period-doubling bifurcation can be derived from very simple considerations on the Poincare 
map. The general features of this influence are, first, an amplification of both noise and signal as the bifurcation point is approached, and second, a stabilization of the dynamical system against the bifurcation. The shift of the bifurcations point is proportional to the squared amplitude of the perturbation. Agreement between numerical studies on the microwave-driven Josephson junction and the above theory is found.
${ }^{1}$ K. Wiesenfeld and B. McNamara, Phys. Rev. Lett. 55, 10 (1985).

${ }^{2}$ K. Wiesenfeld and B. McNamara, Phys. Rev. A 33, 629 (1986).

${ }^{3}$ P. Bryant and K. Wiesenfeld, Phys. Rev. A 33, 2525 (1986).

${ }^{4}$ K. Wiesenfeld, J. Stat. Phys. 38, 1071 (1985).

${ }^{5}$ H. Svensmark, J. Bindslev Hansen, and N. F. Pedersen, Phys. Rev. A 35, 1457 (1987).

6J. Heldstab, H. Thomas, T. Geisel, and G. Radons, Z. Phys. B 50, 141 (1983).

${ }^{7}$ G. F. Eriksen, H. Svensmark, J. Bindslev Hansen, N. F. Peder- sen, and M. R. Samuelsen (unpublished).

${ }^{8} \mathbf{J}$. Guckenheimer and P. Holmes, Nonlinear Oscillations, Dynamical Systems and Bifurcations of Vector Fields (Springer, New York, 1984).

${ }^{9}$ For a good description of Josephson junction equations see, for example, the book A Barone and G. Paterno, Physics and Applications of the Josephson Effect (Wiley, New York, 1982).

${ }^{10}$ Giancarlo Benettin, Luigi Galgani, and Jean-Marie Strelcyn, Phys. Rev. A 14, 2338 (1976). 